\title{
Effect of continuous positive airway pressure on allergic rhinitis in patients with obstructive sleep apnea-hypopnea syndrome
}

This article was published in the following Dove Press journal:

Therapeutics and Clinical Risk Management

\author{
Qintai Yang' \\ Hui $\mathrm{Li}^{2}, *$ \\ Weijun $\mathrm{Wu}^{2, *}$ \\ Xuekun Huang' \\ Bo Tu² \\ Yukun $\mathrm{Ma}^{2}$ \\ Huiyi Deng' \\ Meijiao Li' \\ Hongfeng $\mathrm{He}^{2}$ \\ Can $\mathrm{Li}^{2}$ \\ Yongqi Yang ${ }^{2}$ \\ 'Department of Otorhinolaryngology - \\ Head and Neck Surgery, The Third \\ Affiliated Hospital of Sun Yat-sen \\ University, Guangzhou, People's \\ Republic of China; ${ }^{2}$ Department of \\ Otorhinolaryngology - Head and Neck \\ Surgery, The First Affiliated Hospital of \\ Jinan University, Guangzhou, People's \\ Republic of China \\ *These authors contributed equally \\ to this work
}

Background: The effect of continuous positive airway pressure (CPAP) on nasal symptoms in patients with obstructive sleep apnea-hypopnea syndrome (OSAHS) remains controversial. In addition, possible mechanism(s) remains unclear.

Objective: To examine whether CPAP contributes to the onset and exacerbation of allergic rhinitis (AR) in OSAHS patients based on patient-reported changes in nasal symptoms with and without CPAP therapy and the detection of house dust mites (HDMs) in CPAP filters and household environments.

Materials and methods: In a cohort of OSAHS patients, 350 patients who were undergoing CPAP therapy and 100 patients who declined CPAP therapy were retrospectively analyzed. These patients were contacted by telephone and asked to compare their nasal symptoms before and after CPAP therapy. HDMs in air conditioner and CPAP device filters and in household dust samples were detected using enzyme-linked immunosorbent assays.

Results: The prevalence of AR was higher in the CPAP group vs the non-CPAP group $(15.8 \%$ vs $7.0 \%$, respectively; $P=0.025$ ). The onset of AR among the OSAHS patients with no previous history of AR significantly increased within the first year of CPAP therapy compared with the control group $(5.7 \%$ vs $0 \%$, respectively; $P=0.031)$. Meanwhile, the patients with a history of AR were more likely to experience exacerbated rhinitis symptoms within the second year of treatment compared with the control group $(7.5 \%$ vs $0 \%$, respectively; $P=0.005)$. There was no significant difference in HDM concentrations between the CPAP and air conditioner filters, yet the concentrations in both the filters were higher than the concentrations in the bedroom dust samples (all $P<0.05$ ).

Conclusion: CPAP is associated with the onset and exacerbation of AR in OSAHS patients. Long-term exposure to high concentrations of HDM allergens may be a significant factor.

Keywords: obstructive sleep apnea-hypopnea syndrome, continuous positive airway pressure, allergic rhinitis, house dust mites, allergens

\section{Introduction}

Obstructive sleep apnea-hypopnea syndrome (OSAHS) involves recurrent episodes of upper airway collapse during sleep. These episodes result in hypopnea or apnea, and they are often associated with oxygen desaturation and arousal from sleep. It has been hypothesized that alternating between tissue hypoxia and wakening from sleep may stimulate the sympathetic nervous system, thereby inducing a series of cascading reactions and possibly increasing the risk of cardiovascular disease. ${ }^{1}$

Currently, the preferred treatment for OSAHS is continuous positive airway pressure (CPAP). Application of CPAP can, to some extent, reverse the changes
Correspondence: Qintai Yang Department of Otorhinolaryngology Head and Neck Surgery, The Third Affiliated Hospital of Sun Yat-sen University, 600 Tianhe Road, Guangzhou 510630, People's Republic of China Tel/fax +86 2085252239

Email yang.qt@।63.com 
in pathophysiology that are associated with OSAHS and improve clinical symptoms. ${ }^{2,3}$ However, in some patients, CPAP can contribute to nasal symptoms, thereby leading to reduced compliance with CPAP therapy. According to a survey conducted by Wang et al in Guangzhou, People's Republic of China, the rate of compliance with CPAP therapy was found to be $54.8 \% .^{4}$ Other studies have demonstrated that rhinitis is a risk factor for OSAHS patients and it affects the compliance with CPAP treatment.

In a short-term study involving the application of CPAP with a nasal mask, both nasal inflammation and nasal symptoms were observed. ${ }^{5,6}$ In other studies, concentrations of house dust mites (HDMs) were found to be related to the incidence of allergic disease symptoms, and in Guangzhou, People's Republic of China, HDMs have been identified as the main allergen. ${ }^{7,8}$ It is possible that continuous pressure injury due to CPAP can lead to damage to the junction of nasal mucous cells and the natural membrane barrier, thereby making affected individuals more sensitive to HDMs. To explore whether CPAP contributes to the onset or exacerbation of allergic rhinitis (AR), and also whether HDMs play a role, we retrospectively investigated alterations in nasal symptoms after CPAP therapy among a population of OSAHS patients who were diagnosed in our sleep center. Concentrations of HDMs were also determined in CPAP filters, air conditioner filters, and household dust samples for this cohort.

\section{Materials and methods Patient selection}

This study was approved by the review board of The Third Affiliated Hospital of Sun Yat-sen University, Guangzhou, People's Republic of China, and written informed consent was obtained from all the patients.

Initially, a total of 350 OSAHS patients who were diagnosed at the Department of Sleep Center of The Third Affiliated Hospital of Sun Yat-sen University (Guangzhou, People's Republic of China) between January 2013 and December 2016 were identified. Males and females older than 40 years with moderate-to-severe OSAHS (eg, those with an apnea-hypopnea index value $\geq 15$ ) who lived in Guangzhou, People's Republic of China, were eligible for this study. Patients were excluded if a severe, decompensated comorbid or systemic disease was present, if nasal diseases that could interfere with the observation of rhinitis caused by CPAP were present (eg, nasal surgery, sinonasal inflammation, seasonal rhinitis, etc.), and/or if cognitive impairment that could limit comprehension or participation in the study were observed. In addition, patients who missed more than two weeks of CPAP therapy or those who did not respond to contact by telephone were excluded.

A total of 316 patients met the inclusion criteria for this study, and both their baseline and recent nasal symptom evaluations were retrospectively assessed to obtain a nasal symptom score. An additional 100 OSAHS patients who were diagnosed in the same department between January 2013 and December 2013 and who refused CPAP therapy due to lack of comfort with the therapy, unwillingness to undergo the therapy, and/or economic reasons were included as a control group. A preestablished questionnaire was used to compare demographic variables of these two groups. This questionnaire was completed via telephone and included items that addressed previous history of allergic diseases and/or rhinitis, nasal symptoms before and after CPAP, severity of OSAHS, body mass index (BMI), and complications and current CPAP situation.

\section{Skin prick test}

Both the CPAP and non-CPAP patients with rhinitis symptoms were offered a skin prick test as an outpatient procedure to determine their sensitivity to HDMs. These patients were asked to discontinue their use of antihistamine drugs one week prior to testing. Histamine $(1.70 \mathrm{mg} / \mathrm{mL})$ and a diluent were used as positive and negative controls, respectively. A positive reaction was recorded if a wheal area was greater than $25 \%$ of the positive control and greater than the negative control after $20 \mathrm{~min}$. All reagents were available as part of a standard HDMs allergen extract panel (WOLWO, Huzhou, People's Republic of China).

\section{Nasal symptom scores}

Nasal symptom scores were recorded according to the following scale of nasal symptoms: $0=$ no symptoms; $1=$ mild symptoms (symptoms clearly present, but minimal awareness and easily tolerated); 2 = moderate symptoms (definite awareness of bothersome, yet tolerable, symptoms); and $3=$ severe symptoms (hard-to-tolerate symptoms that interfered with daily living and/or sleeping activities). ${ }^{6,9}$

\section{Collection of dust samples}

All of the study participants had been living in their homes for at least one year prior to the collection of dust samples. In addition, it was requested that the filters would not be replaced for three months prior to the collection of the samples. For 10 of the OSAHS patients who were undergoing CPAP, a brush was used to gently collect dust samples from 
air conditioner and CPAP device filters in their household. Because the size of CPAP device filters was too small to collect with vacuum, which was better for mite collection, ${ }^{10}$ all dust samples were collected with brushes. Dust samples were also collected from the floor of the patients' bedrooms according to the same method.

Large particles were removed from the dust samples prior to their extraction with $0.125 \mathrm{~mol} / \mathrm{L} \mathrm{NH}_{4}\left(\mathrm{HCO}_{3}\right)(1: 15$ dust to buffer, $\mathrm{w} / \mathrm{v}$ ) for $2 \mathrm{~h}$ at room temperature with gentle shaking. The extract solutions were subsequently applied to $0.22 \mu \mathrm{m}$ filters and stored at $-18^{\circ} \mathrm{C}$.

\section{Enzyme-linked immunosorbent assay (ELISA)-based HDM test}

ELISAs were performed to detect allergens present in the extracted dust samples as described previously. ${ }^{11}$ A monoclonal antibody recognizing the HDM allergen, Der f 1 (INDOOR Biotechnologies Limited, Charlottesville, VA, USA), was used. ELISA data were reported in micrograms per gram of dust.

\section{Statistical analyses}

Categorical variables are expressed as frequencies and percentages, and quantitative variables are expressed as mean \pm SD. Proportions were compared by using the Chisquare test or Fisher's exact test, while quantitative variables were compared by using Student's $t$-test or the MannWhitney $U$-test, depending on whether normal or abnormal distribution of data was observed. A logistic regression analysis was performed to assess the possible influence of changes in nasal symptoms. Allergen levels were expressed as median and interquartile range values. The Mann-Whitney $U$-test was used to compare allergen levels among the groups.
Statistical analyses were performed with SPSS version 13.0 software (SPSS Inc., Chicago, IL, USA). $P$-values less than 0.05 were considered statistically significant.

\section{Results}

A total of 416 OSAHS patients met the inclusion criteria for this study, including 316 patients who were receiving CPAP therapy and 100 patients who did not accept CPAP therapy. The latter group served as the control group. All OSAHS patients used modern CPAP device which comes with a heated humidifier and an automated pressure regulator. To accommodate CPAP, they usually selected nasal mask. Various characteristics were compared for the CPAP and non-CPAP groups (summarized in Table 1). There were no significant differences between the two groups at baseline. In particular, the percentage of patients with a history of AR was similar between the two groups. Both groups also exhibited a high prevalence of complications due to cardiovascular disease (38.3\% and $37.0 \%$, respectively).

In the multivariate analysis that was performed, adequate compliance of CPAP use, low frequency of changing filters, and a history of allergic diseases were identified as independent factors related to an increase in nasal symptom score. In contrast, patient age, gender, BMI, comorbidities, and severity of OSAHS were not associated with an increase in nasal symptom score (Table 2).

A total of 316 OSAHS patients were administered CPAP over one year. Subsequently, 200 of these patients maintained CPAP therapy for a total of two years, and 98 patients maintained CPAP therapy for more than three years. The patients without a history of rhinitis who received CPAP therapy exhibited a significantly higher prevalence of rhinitis symptoms within their first year of CPAP treatment compared

Table I Characteristics of OSAHS patients with and without CPAP therapy

\begin{tabular}{|c|c|c|c|}
\hline & $\operatorname{CPAP}(n=316)$ & Non-CPAP $(n=100)$ & $P$-value \\
\hline Age (years), mean \pm SD & $55.21 \pm 8.0$ & $54.36 \pm 7.5$ & 0.349 \\
\hline Gender, male/female & $261 / 55$ & $85 / 15$ & 0.575 \\
\hline BMI $\left(\mathrm{kg} / \mathrm{m}^{2}\right)$, mean $\pm \mathrm{SD}$ & $26.76 \pm 2.93$ & $27.19 \pm 2.96$ & 0.195 \\
\hline \multicolumn{4}{|l|}{$\mathrm{AHI}$, mean $\pm \mathrm{SD}$} \\
\hline Moderate & $23.89 \pm 4.42$ & $23.09 \pm 4.49$ & 0.288 \\
\hline Severe & $47.16 \pm 7.62$ & $48.44 \pm 8.27$ & 0.290 \\
\hline Severity of OSAHS (moderate/severe) & $77.5 \%(138 / 178)$ & $85.2 \%(46 / 54)$ & 0.683 \\
\hline \multicolumn{4}{|l|}{ Complication } \\
\hline Cardiovascular disease & $38.3 \%(|2| / 3 \mid 6)$ & $37.0 \%(37 / 100)$ & 0.817 \\
\hline Diabetes & $16.8 \%(53 / 316)$ & $1 \mathrm{I} .0 \%(\mathrm{II} / \mathrm{I00})$ & 0.163 \\
\hline Neurocognitive effects & $12.0 \%(38 / 3 \mid 6)$ & $13.0 \%(13 / 100)$ & 0.796 \\
\hline AR history & $8.2 \%(26 / 316)$ & $6.0 \%(6 / 100)$ & 0.466 \\
\hline
\end{tabular}

Abbreviations: OSAHS, obstructive sleep apnea-hypopnea syndrome; CPAP, continuous positive airway pressure; SD, standard deviation; BMI, body mass index; $\mathrm{AHI}$, apnea-hypopnea index; AR, allergic rhinitis. 
Table 2 Logistic regression analysis of risk factors that induce an increase in nasal symptom score after CPAP therapy

\begin{tabular}{llll}
\hline Risk factors & OR & $\mathbf{9 5 \% ~ C l}$ & $P$-value \\
\hline Age & 0.73 & $0.44-1.22$ & 0.228 \\
Gender & 1.06 & $0.41-2.76$ & 0.900 \\
BMI & 1.22 & $0.73-2.02$ & 0.452 \\
Severity of OSAHS & 1.73 & $0.79-3.78$ & 0.170 \\
Complication & 1.23 & $0.92-1.65$ & 0.167 \\
$\begin{array}{l}\text { CPAP compliant (four or } \\
\text { more hours a day) }\end{array}$ & 2.53 & $1.05-6.13$ & 0.040 \\
$\begin{array}{l}\text { Frequency of filter change } \\
\text { (less than four times a year) }\end{array}$ & 2.87 & $1.35-6.11$ & 0.006 \\
$\begin{array}{l}\text { History of allergic diseases } \\
\text { A }\end{array}$ & 21.92 & $9.21-52.17$ & 0.000 \\
\hline
\end{tabular}

Abbreviations: CPAP, continuous positive airway pressure; OR, odds ratio; $\mathrm{Cl}$, confidence interval; BMI, body mass index; OSAHS, obstructive sleep apnea-hypopnea syndrome.

to the control group $(5.7 \%$ vs $0 \%$, respectively; $P=0.031)$. On the other hand, the patients with a history of rhinitis who received CPAP therapy exhibited a significantly higher prevalence of exacerbation of their nasal symptoms within the second year of CPAP treatment compared to the control group $(7.5 \%$ vs $0 \%$, respectively; $P=0.005)$, and this was not observed within their first year of CPAP treatment.

The percentage of AR patients increased from $8.2 \%$ to $15.8 \%$ in the CPAP group, while it only increased from $6.0 \%$ to $7.0 \%$ in the non-CPAP group. Significant differences in AR morbidity between the CPAP and non-CPAP groups were also observed after CPAP therapy $(15.8 \%$ vs $7.0 \%$, respectively; $P=0.025$ ) (Table 3 ).

There were no significant differences in the concentration of HDMs that were measured in the air conditioner filter vs CPAP device filter (mean rank: 9.7 vs 11.3, respectively; $P=0.579$ ). However, HDM allergen levels were significantly

Table 3 Nasal symptoms after CPAP therapy

\begin{tabular}{|c|c|c|c|}
\hline Parameter examined & CPAP & Non-CPAP & $P$-value \\
\hline \multicolumn{4}{|l|}{ Onset of nasal symptom } \\
\hline Within one year & $5.7 \%(18 / 316)$ & $0(0 / 100)$ & 0.031 \\
\hline Within one to two years & $2.5 \%(5 / 200)$ & $1.0 \%(1 / 100)$ & 0.662 \\
\hline Within two to three years & $1.0 \%(1 / 98)$ & $0(0 / 100)$ & 0.495 \\
\hline HDM skin prick test & $100 \%(24 / 24)$ & $100 \%(1 / 1)$ & 1.000 \\
\hline \multicolumn{4}{|l|}{ Exacerbation of nasal symptom } \\
\hline Within one year & $0.6 \%(2 / 316)$ & $0(0 / 100)$ & 1.000 \\
\hline Within one to two years & $7.5 \%(15 / 200)$ & $0(0 / 100)$ & 0.005 \\
\hline Within two to three years & $3.1 \%(3 / 98)$ & $0(0 / 100)$ & 0.238 \\
\hline HDM skin prick test & $100 \%(20 / 20)$ & - & - \\
\hline Nasal symptom unchanged & $1.9 \%(6 / 316)$ & $6.0 \%(6 / 100)$ & 0.073 \\
\hline HDM skin prick test & $100 \%(6 / 6)$ & $100 \%(6 / 6)$ & 1.000 \\
\hline AR incidence & $15.8 \%(50 / 3 \mid 6)$ & $7.0 \%(7 / 100)$ & 0.025 \\
\hline
\end{tabular}

Note: HDM skin prick test indicates the percent of positive result.

Abbreviations: CPAP, continuous positive airway pressure; HDM, house dust mite; $A R$, allergic rhinitis. higher in the CPAP filter samples compared to the bedroom samples (mean rank: 15.5 vs 5.5 , respectively; $P=0.000$ ) (Figure 1). Among the patients who underwent a skin prick test to detect sensitivity to HDMs, all received a positive result.

\section{Discussion}

Among the OSAHS patients who were examined, a relationship between CPAP therapy and onset or exacerbation of AR was observed. We hypothesize that long-term exposure to high concentrations of HDM allergens is responsible for this result.

Currently, CPAP is the preferred treatment for OSAHS patients. However, while this therapy is beneficial, it is associated with a low level of adherence, partly due to the side effects of CPAP. In our clinic, we observed that a subset of OSAHS patients who received CPAP treatment developed AR or exhibited an exacerbation of AR. Therefore, the aim of this study was to explore a possible relationship between CPAP and AR, and to identify a possible underlying mechanism. Thus, 416 OSAHS patients were retrospectively analyzed, including 316 patients undergoing CPAP therapy and 100 patients who declined CPAP therapy. In the former group, the OSAHS patients tended to have rhinitis symptoms and an increase in nasal symptom scores compared with the non-CPAP group. When an HDM skin prick test was conducted in the patients with rhinitis symptoms from both groups, all of them had a positive reaction. Morbidity due to AR was also almost two-fold higher in the CPAP group compared to the non-CPAP group and the general population of Guangzhou. ${ }^{12}$ Meanwhile, the prevalence of AR at baseline between the two groups did not significantly differ, and it was similar to the reported prevalence of AR in Guangzhou. ${ }^{12}$ Therefore, the results of the present study suggest that CPAP therapy may increase the incidence of AR.

Previously published data regarding a relationship between CPAP and rhinitis have been inconsistent. For example, while most of the studies have revealed an infiltration of neutrophils into inflamed nasal mucous membrane following CPAP treatment of OSAHS patients, ${ }^{6}$ not all of the studies have observed the same rhinitis symptoms. ${ }^{5,6,13,14}$ These differences may be due to the methodologies used. For the prospective studies that have been conducted, these have rarely extended over more than one year due to the difficulties associated with long-term follow-up studies. In the present study, the follow-up period extended up to three years for a subset of the patients, and a higher rate of onset or exacerbation of rhinitis symptoms was observed in the CPAP group compared with the non-CPAP group. 


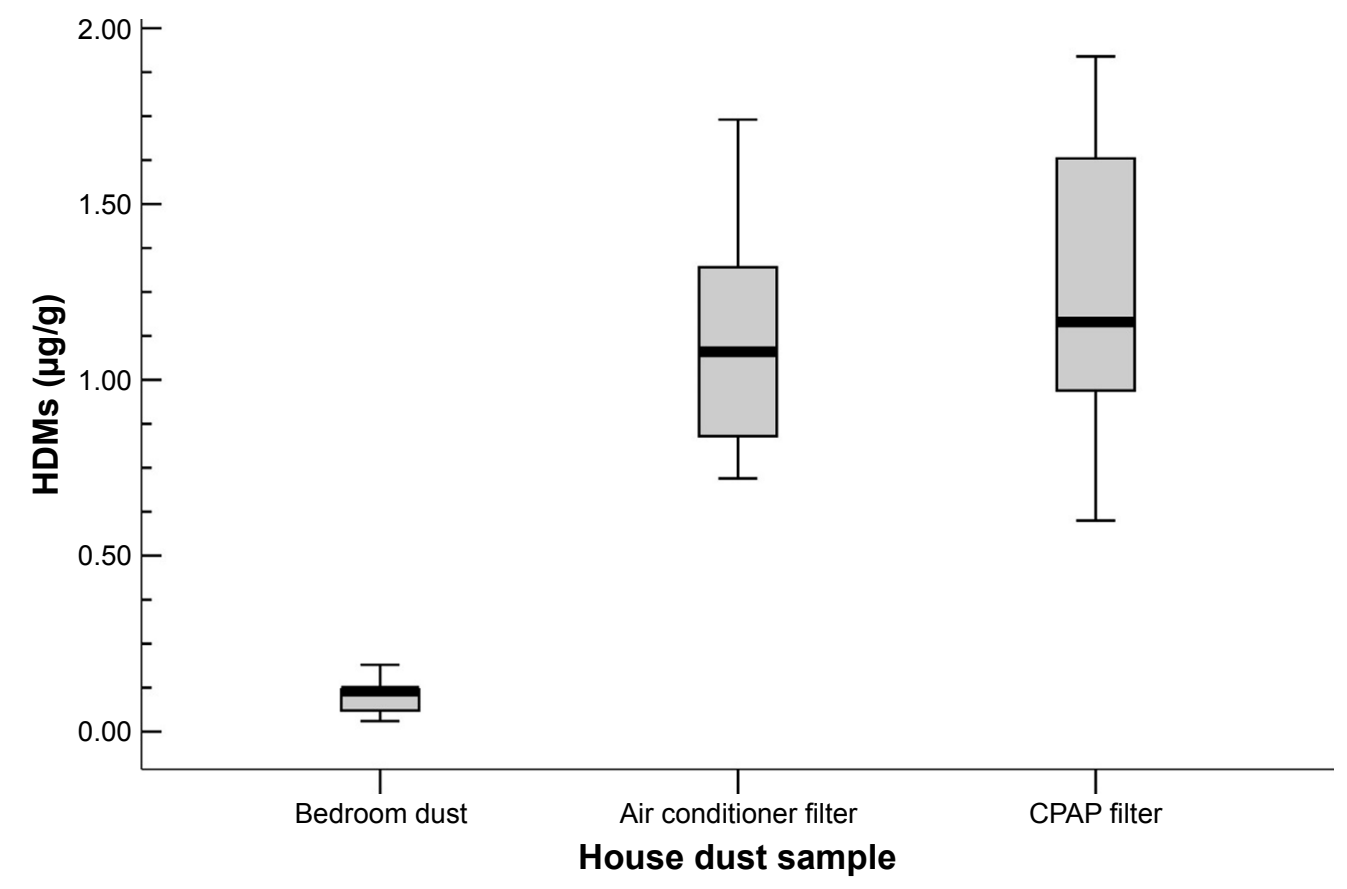

Figure I Differences in HDM concentrations among the groups.

Abbreviations: HDM, house dust mite; CPAP, continuous positive airway pressure.

An interesting observation was made regarding the role of AR in relation to CPAP therapy. Among the OSAHS patients without a history of AR, a significant increase in the onset of AR was observed during the first year of CPAP therapy. Meanwhile, among the OSAHS patients with a history of $A R$, a significant increase in the exacerbation of rhinitis symptoms was observed during the second year of CPAP treatment. Similarly, Cisternas et $\mathrm{al}^{6}$ observed an exacerbation of rhinitis symptoms in OSAHS patients without AR after two months of CPAP treatment, and no exacerbation of rhinitis symptoms has been observed in OSAHS patients with AR. The authors who reported the latter result proposed that a protective mechanism may exist in AR patients which prevents a second stimulation of the nose. Based on this possibility, we hypothesize that a protection mechanism exists in AR patients whereby an exacerbation of rhinitis symptoms is prevented during its early stages. We have also considered that longer CPAP treatment periods may damage the junctions of nasal mucous cells and the natural membrane barrier, thereby facilitating alterations in nasal symptoms. Further studies are needed to validate these possibilities.

It has been shown that CPAP treatment leads to an increase in nasal inflammation, and this may worsen nasal symptoms over time. ${ }^{5,6}$ Nevertheless, the mechanism(s) involved remains unclear. Previous research has demonstrated that cold, dry air can stimulate a release of nasal inflammatory mediators, ${ }^{15,16}$ and use of a supplementary heated humidifier can treat the side effects caused by a conventional CPAP device. ${ }^{17-19}$ However, for subjects without a history of nasal symptoms, exposure to cold, dry air does not result in changes in nasal inflammation mediators and symptoms. ${ }^{15,16}$ In addition, modern CPAP devices are usually accompanied by a heated humidifier. In Guangdong, which is located in the southern region of the People's Republic of China, warmer weather conditions are observed compared with other regions most of the year. ${ }^{20}$ Thus, the challenge presented by cold, dry air may not be the main reason for the observed phenomena. In the multivariate analysis we performed, exacerbation of nasal symptoms was found to be closely related to a history of allergic diseases and the rate of filter replacement for CPAP devices. In a previous study, Wang et $\mathrm{al}^{7}$ found that HDMs were the predominant aeroallergen in Guangzhou, and this incidence ranged from $68.5 \%$ to $83.2 \%$ in AR patients who were older than 40 years. On the other hand, HDM-sensitive AR patients usually experience a relapse when air conditioners are used in summer. A high level of Dermatophagoides farinae 1 has been found in air conditioner filters, ${ }^{20}$ and reduced exposure to HDMs has been shown to result in an improvement in clinical symptoms. ${ }^{8}$ Thus, we speculate that a relationship exists between the HDM concentration in CPAP devices and the incidence of AR.

To investigate a possible connection between AR and HDMs in CPAP devices, dust samples were randomly collected 
from air conditioner and CPAP device filters which had not been cleaned for three months, and also from the bedrooms of OSAHS patients in Guangzhou. Based on the ELISA results obtained, there was no significant difference in the concentration of HDMs between the air conditioner and CPAP device filters. However, the levels of HDM allergens were significantly higher in the filter samples compared to the bedroom samples. These results imply that OSAHS patients who are undergoing CPAP treatment are exposed to a high concentration of allergens when they use CPAP devices, and this exposure has the potential to induce the onset of AR, or exacerbate an AR condition.

To our knowledge, this is the first study to explore the concentration of HDMs in CPAP devices. However, it is important to note some considerations when examining these data. First, due to the retrospective design of this study, it was difficult to determine the exact relationship between CPAP therapy and AR over an extended period of time. Thus, a multicenter, randomized, and controlled trial should be conducted with a large patient sample. Second, only HDM allergens were detected, and there are other allergens which have also been shown to induce AR. Therefore, these additional allergens also need to be investigated in relation to CPAP therapy. Moreover, objective measuring is also important, and eosinophil count in nasal secretions of subjects should be measured to exclude local allergic nasal reactions. Finally, it is reported that pressure of CPAP device has an effect on the nasal inflammation. However, because modern CPAP device that our patients used comes with an automated pressure regulator and patients' physical condition changes over time, which has an influence on the pressure of CPAP, we cannot obtain exact data on CPAP pressure and evaluate its effect on AR.

\section{Conclusion}

The results of this study demonstrate that onset or exacerbation of AR is directly related to CPAP treatment in patients with OSAHS. It remains for further investigations to determine the clinical relevance of this finding, although we hypothesize that a high concentration of HDMs in CPAP device filters contributes to the incidence/exacerbation of AR. In the meantime, it is important that clinicians closely monitor evaluations and the health education of patients with nasal diseases, especially AR, particularly among those with OSAHS undergoing CPAP therapy. It is also advised that CPAP device filters should be changed frequently, thereby minimizing patient exposure to HDM allergens.

\section{Acknowledgment}

The authors thank all the participants of this study. This paper has not been published elsewhere in whole or in part.

\section{Disclosure}

The authors report no conflicts of interest in this work.

\section{References}

1. Javaheri S, Barbe F, Campos-Rodriguez F, et al. Sleep apnea: types, mechanisms, and clinical cardiovascular consequences. J Am Coll Cardiol. 2017;69(7):841

2. Patel SR, White DP, Malhotra A, et al. Continuous positive airway pressure therapy for treating sleepiness in a diverse population with obstructive sleep apnea: results of a meta-analysis. Arch Intern Med. 2003;163(5):565-571.

3. Giles TL, Lasserson TJ, Smith BH, et al. Continuous positive airways pressure for obstructive sleep apnoea in adults. Cochrane Database Syst Rev. 2006;6(2):CD001106.

4. Wang Q, Ou Q, Tian XT, et al. 阻塞性睡眠呼吸暂停患者持续气道 正压通气治疗的长期依从性 [Analysis of long-term compliance to continuous positive airway pressure in patients with obstructive sleep apnea]. Zhonghua Yi Xue Za Zhi. 2016;96(30):2380. Chinese [with English abstract].

5. Vilaseca I, Lehrer-Coriat E, Torres M, et al. Early effects of continuous positive airway pressure in a rodent model of allergic rhinitis. Sleep Med. 2016;27:25-27.

6. Cisternas A, Aguilar F, Montserrat JM, et al. Effects of CPAP in patients with obstructive apnoea: is the presence of allergic rhinitis relevant? Sleep Breath. 2017;21(4):893-900.

7. Wang W, Huang X, Chen Z, et al. Prevalence and trends of sensitisation to aeroallergens in patients with allergic rhinitis in Guangzhou, China: a 10-year retrospective study. BMJ Open. 2016;6(5):e011085.

8. Harving H, Korsgaard J, Dahl R. Clinical efficacy of reduction in house dust mite exposure in specially designed, mechanically ventilated "healthy" homes. Allergy. 1994;49(10):866-870.

9. Pfaar O, Demoly P, Gerth van Wijk R, et al. Recommendations for the standardization of clinical outcomes used in allergen immunotherapy trials for allergic rhinoconjunctivitis: an EAACI Position Paper. Allergy. 2014;69(7):854-867.

10. Abbott J, Cameron J, Taylor B. House dust mite counts in different types of mattresses, sheepskins and carpets, and a comparison of brushing and vacuuming collection methods. Clin Exp Allergy. 1981;11(6):589-595.

11. Zheng YW, Lai XX, De Yu Z, et al. Indoor allergen levels and household distributions in nine cities across China. Biomed Environ Sci. 2015;28(10):709-717.

12. Zhang Y, Zhang L. Prevalence of allergic rhinitis in China. Allergy Asthma Immunol Res. 2014;6(2):105-113.

13. Skoczyński S, Ograbek-Król M, Tazbirek M, et al. Short-term CPAP treatment induces a mild increase in inflammatory cells in patients with sleep apnoea syndrome. Rhinology. 2008;46(2):144.

14. Balsalobre L, Pezato R, Gasparini H, et al. Acute impact of continuous positive airway pressure on nasal patency. Int Forum Allergy Rhinol. 2017;7(7):712-717.

15. Togias AG, Naclerio RM, Proud D, et al. Nasal challenge with cold, dry air results in release of inflammatory mediators. Possible mast cell involvement. J Clin Investig. 1985;76(4):1375-1381.

16. Togias AG, Naclerio RM, Peters SP, et al. Local generation of sulfidopeptide leukotrienes upon nasal provocation with cold, dry air. $\mathrm{Am}$ Rev Respir Dis. 1986;133(6):1133-1137.

17. Mador MJ, Krauza M, Pervez A, et al. Effect of heated humidification on compliance and quality of life in patients with sleep apnea using nasal continuous positive airway pressure. Chest. 2005;128(4):2151-2158. 
18. Neill AM, Wai HS, Bannan SP, et al. Humidified nasal continuous positive airway pressure in obstructive sleep apnoea. Eur Respir J. 2003;22(2): 258-262.

19. Rakotonanahary D, Pelletier-Fleury N, Gagnadoux F, et al. Predictive factors for the need for additional humidification during nasal continuous positive airway pressure therapy. Chest. 2001;119(2):460-465.
20. Zhang C, Gjesing B, Lai X, et al. Indoor allergen levels in Guangzhou city, southern China. Allergy. 2011;66(2):186-191.

\section{Publish your work in this journal}

Therapeutics and Clinical Risk Management is an international, peerreviewed journal of clinical therapeutics and risk management, focusing on concise rapid reporting of clinical studies in all therapeutic areas, outcomes, safety, and programs for the effective, safe, and sustained use of medicines. This journal is indexed on PubMed Central, CAS,
EMBase, Scopus and the Elsevier Bibliographic databases. The manuscript management system is completely online and includes a very quick and fair peer-review system, which is all easy to use. Visit http://www.dovepress.com/testimonials.php to read real quotes from published authors.

Submit your manuscript here: http://www.dovepress.com/therapeutics-and-clinical-risk-management-journal 